\title{
Weight in Infancy and Obesity in Children Born Preterm
}

\author{
Tetiana Litvinchuk, Ruchi Singh, Christopher T. Sheehan and Tetyana L. Vasylyeva*
}

Department of Pediatrics, Texas Tech University Health Sciences Center at Amarillo, Texas, USA

\begin{abstract}
Excessive weight gain in infancy may lead to obesity and its sequelae later in life. Children born preterm have higher associated risk of becoming obese than full term babies. The goal of the study was to examine early weight gain among preterm infants, who later in life became obese. In a retrospective chart review of 37 preterm infants, 27 grew to support a normal weight and 10 became obese. We demonstrated differences in early childhood weight gain dynamics with weight differences between groups noted at 6 months of age that persisted later in life. Increased risk of obesity could be identified very early in infancy among preterm children. Early nutritional consult and attention to weight gain in infancy and early childhood are important steps in obesity prevention.
\end{abstract}

Keywords: Obesity, Prematurity, Low birth weight, Postnatal care.

\section{INTRODUCTION}

Over one-third of all children and adolescents were overweight or obese in the US in 2012 [1]. The Bogalusa Heart Study showed that children who became obese as early as age 2 were more likely to be obese as adults [2]. Society and healthcare providers now recognize the critical short- and long-term impacts of obesity on the health of the population. Prevention of adult diseases is rooted in pediatric care and preterm babies might be prone to obesity in adulthood [3].

Prematurity is a significant medical problem, which affects nearly 500,000 babies each year in the United States. That is 1 of every 8 infants born in the United States [4]. Preterm babies may develop prematurity-related complications including breathing problems, feeding difficulties, cerebral palsy, and developmental delays. Improvements in neonatal care have led to significant increases in surveillance of preterm babies, and today's challenge for pediatricians is to provide the best possible care to ensure a healthy lifestyle for these children.

The objectives of our study were to identify weight gain dynamics and the earliest age in preterm children when differences in weight-for-length exists between children who became obese or nonobese in adolescence.

"Address corresponding to Tetyana L. Vasylyeva, Texas Tech University Health Sciences Center, School of Medicine, Department of Pediatrics, 1400 Coulter Street, Amarillo, TX 79106, USA; Tel: 806354-5432; E-mail: tetyana.vasylyeva@ttuhsc.edu

\section{METHODS}

A retrospective chart review of 37 Texas Tech University Health Sciences Center at Amarillo Pediatric Clinic patients was conducted. All charts from the clinic belonging to children between 1018 years old who were born at 37 or fewer weeks of gestational age were screened. Charts were pulled by electronic records using the ICD-9 code for prematurity.

Only charts, which contained all well-child visits between birth and 24-months-old and had all appropriate anthropometrical and demographic data were reviewed and included in the study. Maternal, family, neonatal, and postnatal data were abstracted. The child's postnatal and childhood data included type of feeding, major illnesses, blood pressure, weight, and height. Weight-for-length data as a percentile of a normal curve from Centers of Disease Control and Prevention (CDC) charts database were analyzed for visits between 2- and 24-months-of-age. Preterm low birth weight baby growth charts with sex specific weight-forlength percentiles for a very-low-birth-weight group (< or $=1500 \mathrm{~g}$ ) and a low-birth-weight group (1501-2500 g) were used [5].

BMI was calculated as the patient's weight $(\mathrm{kg}) /$ height $^{2}(\mathrm{~m})$ and children charts from the CDC were used for comparison. Obesity was defined as a BMI at or above the 95th percentile for children of the same age and sex [6]. Among the study group 10 children had obesity and 27 normal weights. Overweight children were not included in the study. 
Children involved in the study did not have cerebral palsy or known congenital cardiac, endocrine, or renal disease. The study protocol was approved by the Amarillo TTUHSC Institutional Review Board.

Statistical analyses were performed using Stata, Version 10. Data were presented as mean (M), standard deviation (SD), and standard error of the mean (SEM). To compare weight gains before 24 months in obese and non-obese groups, a two tailed t-test was used.

\section{RESULTS}

Of the 37 subjects, 27 adolescent subjects had normal weight and 10 were obese. Characteristics of the study groups are presented in Table 1. Children in both groups were comparable in gestational age (33.4 \pm 2.9 weeks in obese group vs. $33.6 \pm 2.3$ weeks in non-obese group) and birth weight $(2030.6 \pm 461.3 \mathrm{~g}$ vs. $2017.8 \pm 565.5 \mathrm{~g}$, respectively) with relatively equal numbers of girls and boys. The number of formula-fed babies was greater among the obese group compared to the non-obese group $(70 \%$ vs. $59 \% ; p<0.01)$.
The dynamic and comparative analysis between weight-for-length growth in the obese and nonobese groups is presented in Table 2 and illustrated in Figure 1. Differences between the two groups became obvious and significant by 6 months-of-age $(47.8 \pm 5.1 \%$ vs. $24.1 \pm 10.0 \%$; $p<$ $0.03)$. The differences remained persistent with the highest gap between the groups at 15months-of-age $(80.0 \pm 11.7 \%$ vs. $43.7 \pm 6.2 \%$; $p<$ $0.0001)$ and at 24 months $(65.1 \pm 8.3$ vs. $38.9 \pm$ $5.9 \% ; p<0.01)$

\section{DISCUSSION}

Monitoring and attention to catch-up growth and nutritional needs of premature babies is critical for obesity prevention. Rapid postnatal weight gain after a period of nutritional restriction is associated with the development of insulin resistance and metabolic syndrome in later life [7]. It is now wellrecognized that preterm babies are prone to have unfavorable long-term health outcomes such as increased risk for central obesity, insulin resistance, impaired glucose tolerance, type 2 diabetes, and cardiovascular disease [8-10]. Pre-term, low birth weight babies may be obese in later life

\section{Table 1: Characteristics of Study Group}

\begin{tabular}{|c|c|c|}
\hline Group Characteristics & $\begin{array}{c}\text { Obese } \\
n=10\end{array}$ & $\begin{array}{c}\text { Normal Weight } \\
n=27\end{array}$ \\
\hline Gestational age (weeks; $M \pm S D$ ) & $33.4 \pm 2.9$ & $33.6 \pm 2.3$ \\
\hline Birth weight (g; weeks; $M \pm S D)$ & $2030.6 \pm 461.3$ & $2017.8 \pm 565.5$ \\
\hline Female (\%) & 48 & 30 \\
\hline Male $(\%)$ & 52 & 70 \\
\hline Feeding & & \\
\hline Formula fed (\%) & 70 & 59 \\
\hline Breast milk fed (\%) & 10 & 15 \\
\hline Combined feeding (\%) & 20 & 26 \\
\hline \multicolumn{3}{|c|}{ Distribution of Study Group Per Gestational Age } \\
\hline Gestational age (we & \multicolumn{2}{|r|}{$\mathrm{n}=37$} \\
\hline $27-30$ & \multicolumn{2}{|r|}{4} \\
\hline $31-34$ & \multicolumn{2}{|r|}{14} \\
\hline $35-37$ & \multicolumn{2}{|r|}{19} \\
\hline
\end{tabular}


Table 2: Dynamic and Comparative Analysis Between Weight-for-Length Growth in Obese and Non-Obese Groups

\begin{tabular}{|cccc|}
\hline Months & Obese & Normal Weight & $\boldsymbol{p}$ Value (Two Tailed) \\
\hline 2 months & 39.25 & 22.97 & 0.09 \\
4 months & 40.11 & 31.37 & 0.348 \\
6 months & 47.88 & 24.13 & $0.03^{* *}$ \\
9 months & 59.25 & 37.38 & $0.05^{*}$ \\
12 months & 61.29 & 35.95 & $0.02^{* *}$ \\
15 months & 80.00 & 43.68 & $0.001^{* * *}$ \\
18 months & 65.62 & 39.34 & $0.05^{*}$ \\
24 months & 65.11 & 38.88 & $0.01^{* *}$ \\
\hline
\end{tabular}

$*<0.05 ; * * 0.01 ; * * * 0.001$.

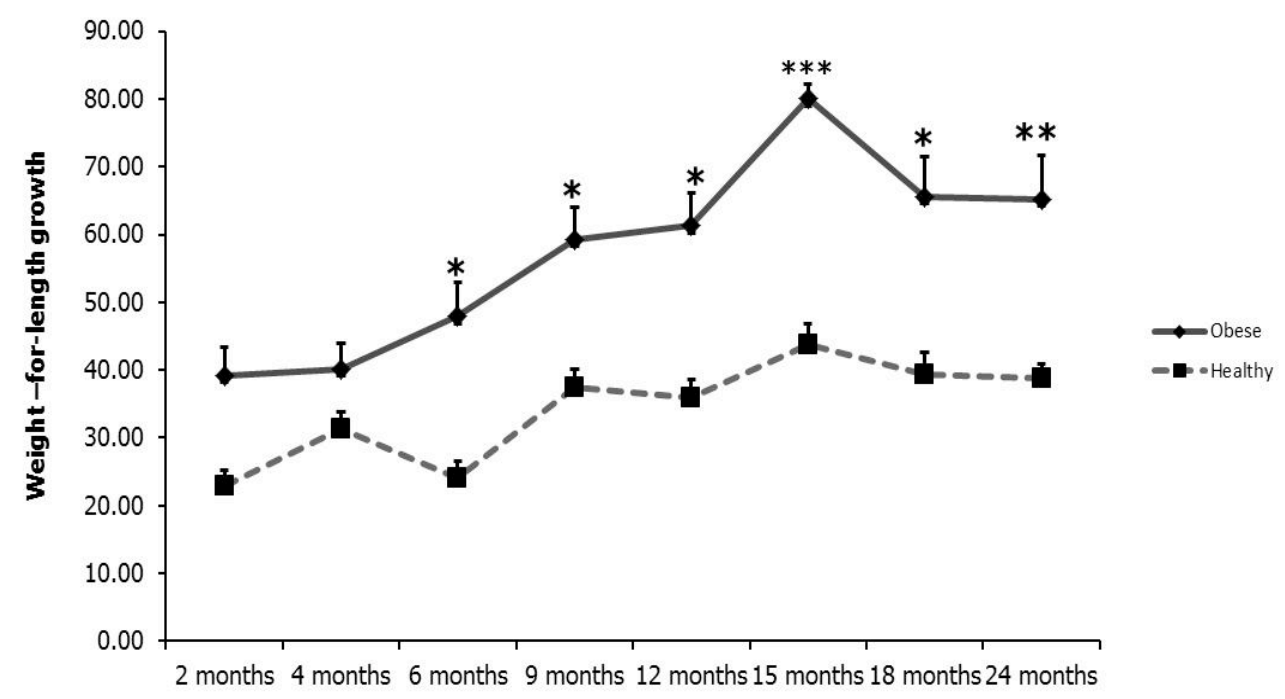

Figure 1: Infant weight gain dynamic between obese and normal weighted adolescents born preterm * $<0.05$; ${ }^{* *} \leq 0.01 ;{ }^{* * *} \leq 0.001$.

[11-14]. Catch-up weight gain between birth and 2 years-of-age was shown to have a dramatic effect upon central adiposity and insulin resistance even at 4-years-old [11].

Our data showed that at 6 months-of-age the difference in average weight among future obese and non-obese groups of children became obvious. At the same time, the individual weight-forlength index, even after correction for gestation age, might look normal in children destined to become obese. That fact makes an individual prediction challenging and more study is needed to give pediatricians a reliable instrument to identify risk of obesity in preterm babies. The charts used reflected current (nutritional) practices, which needs optimization [7]. In our study, we showed how weight differences early in infancy predicted obese and non-obese weights in adolescence. Because it was a retrospective study we were unable to consider the whole spectrum of clinical data, including detailed nutrition and care in the NICU. In addition, we followed well-child visits starting at 2-months-old, although some very low birth weight babies might stay at the NICU beyond this age and were excluded from the study.

Our data confirmed a conclusion made by others that weight gain in the first 6 months is especially predictive of later obesity risk due to the metabolic programming that can occur early postpartum [15]. We are in agreement with the authors that 
the first 6 months are a critical window, and it is true not only for term, but preterm babies.

A significant jump in weight gain between 12 and 15 months seems to occur when children are weaned from formula or breast milk. This is also a critical time to address the concern of obesity in the clinical setting. It corresponds to data of Ahn et al. that showed that the early 'catch-up phenomenon' with an accelerated growth rate occurred around 11-months-of-corrected-age in premature babies [16].

The prevalence of formula-fed babies among the obese preterm children in our cohort is a modifiable factor, which could prevent obesity. These data support Zarrati et al. findings, which showed that breast milk feeding and its long-term consequences were important factors for preventing metabolic syndrome in childhood and later life [17]. Breastfeeding and timely introduction of complimentary foods are widely considered strong protectors against obesity later in life $[18,19]$. More attention is now paid to individual characteristics of breast milk. Thus, human milk high in adeponectin, which is an insulin-sensitizing and anti-inflammatory molecule, affects infants' weight trajectories during the first two years of life [20]. Breast feeding during the first year of life with slow introduction of baby-food might have life-long benefit for preterm babies.

Ong et al. identified a role of leptin in the regulation of infancy weight gain, and suggested a mechanism whereby infants may 'catch-up' in growth postnatally. Interesting recent work showed that higher perinatal leptin was associated with lower 3-year adiposity, whereas higher age 3 leptin was associated with greater weight gain and adiposity in later childhood [21]. Other authors have lower levels of leptin in pre-term infants compared with term newborns [22]. Maternal diet could be an additional modifiable factor affecting levels of leptin in breast-fed babies, and is preferable for preterm infants [23]. The current practice of fortification of expressed breast milk may increase weight gain and skeletal and head growth during infancy [22]. Feeding preterm babies with nutrient-enriched formula following hospital discharge is a common practice. Some evi- dence suggests this may increase growth rates up to 18 months corrected age, but there is no long term outcome data to compare nutrient-enriched formula versus standard formula in premature born babies at older ages [24].

An adverse intrauterine environment is a risk factor for excessive catch up growth [25]. The vast majority of our babies were adequate for gestational age. It is possible, that the approach to early nutrition might be different to those for preterm babies who were born small for gestational age, with intrauterine growth retardation and those who are although small are adequate for gestational age in terms of birth weight. More research is needed on this topic.

A multicenter European study focused on the introduction of solid food and growth in the first 2 years of life in formula-fed children. The study was not centered on preterm babies, but showed that babies who received solid-food during the first 12 weeks of life experienced early catch-up growth, whereas those introduced to solid food at $>22$ weeks of age grew more slowly and stayed on lower trajectories [26]. Unfortunately, no similar high-quality studies have been conducted with preterm babies.

All these findings indicate the need for early vigilance in monitoring and addressing catch-up growth to prevent obesity as a future sequelae. Encouragement of breast feeding, timely changes from high to normal caloric formulas, and nutritional consultation are all easy, but extremely valuable interventions that could prevent obesity in the vulnerable preterm pediatric population. The suggestion of Fanaro to focus on improving the early nutrient management of premature infants in NICU allowing babies to reach an adequate growth rate (at least $18-20 \mathrm{~g} / \mathrm{kg} / \mathrm{d}$ ) to avoid the need of a late unphysiological catch-up growth seems very reasonable [27].

Although we noticed the weight difference between infants who were born preterm and became obese versus those who did not, our study did not identify the best recipe for feeding. Multicenter national studies are needed to define the best approach to nutrition in the NICU and post-discharge for preterm born babies. The results of such stud- 
ies would direct post-hospital discharge nutrition planning for general pediatricians, give reliable instruments to measure nutritional adequacy, and help prevent obesity in individuals born preterm.

\section{CONCLUSION}

Careful monitoring of weight gain may lead to early anticipation of future obesity. High caloric formulas for low birth weight babies and fortification of expressed breast milk should be reconsidered at least by the age of 4-months-old, even if actual percentile on weight-for-length values for preterm babies are normal. Early nutritional consult and attention to weight gain early in life are important steps in obesity prevention.

\section{CONFLICTS OF INTEREST}

There are no conflicts of interest to declare.

\section{REFERENCES}

[1] Ogden CL, Carroll MD, Kit BK, Flegal KM. Prevalence of childhood and adult obesity in the United States, 2011-2012. J Am Med Assoc 2014; 311: 806-14.

[2] Freedman DS, Khan LK, Serdula MK, et al. The relation of childhood BMI to adult adiposity: the Bogalusa Heart Study. Pediatrics 2005; 115: 22-27.

[3] Vasylyeva TL, Barche A, Chennasamudram SP, et al. Obesity in prematurely born children and adolescents: follow up in pediatric clinic. Nutr J 2013; 12: 150.

[4] Division of Reproductive Health, National Center for Chronic Disease Prevention and Health Promotion. Updated December 9, 2013; Cited October 2, 2014: Available from: http://www.cdc.gov/reproductivehealth/MaternallnfantHealth/ PretermBirth.htm.

[5] Guo SS, Wholihan K, Roche AF, Chumlea WC, Casey PH. Weight-for-length reference data for preterm, low-birth-weight infants. Arch Pediatr Adolesc Med 1996; 150: 964-70.

[6] Barlow SE. Expert committee recommendations regarding the prevention, assessment, and treatment of child and adolescent overweight and obesity: Summary report. Pediatrics 2007; 120: 164-92.

[7] Corpeleijn WE, Kouwenhoven SMP, van Goudoever JB. Optimal growth of preterm infants. World Rev Nutr Diet; 2013. 106: 149-55.

[8] Ong KKL, Ahmed ML, Emmett PM, Preece MA, Dunger DB. Association between postnatal catch-up growth and obesity in childhood: prospective cohort study. Brit Med J 2000; 320: 1244.

[9] Forsen T, Eriksson JG, Tuomilehto J, Reunanen A, Osmond $C$, Barker DJP. The fetal and childhood growth of persons who develop type 2 diabetes. Ann Intern Med 2000; 133: 176-82.

[10] Eriksson JG, Forsen T, Tuomilehto J, Jaddoe VWV, Osmond $C$, Barker DJP. Effects of size at birth and childhood growth on the insulin resistance syndrome in elderly individuals. Diabetologia 2002; 45: 342-48.

[11] Ibanez L, Ong K, Dunger DB, de Zegher F. Early development of adiposity and insulin resistance after catchup weight gain in small-for-gestational-age children. J Clin Endocr Metab 2006; 91: 2153-58.

[12] Cottrell EC, Ozanne SE. Early life programming of obesity and metabolic disease. Physiol Behav 2008; 94: 17-28.

[13] Zarrati M, Shidfar F, Razmpoosh E, et al. Does low birth weight predict hypertension and obesity in school children? Ann Nutr Metab 2013; 63: 69-76.

[14] Desai M, Beall M, Ross MG. Developmental origins of obesity: Programmed adipogenesis. Curr Diabetes Rep 2013; 13: 27-33.

[15] Young BE, Johnson SL, Krebs NF. Biological determinants linking infant weight gain and child obesity: current knowledge and future directions. Adv Nutr 2012; 3: 675-86.

[16] Ahn $Y$, Sohn M, Jun $Y$, Lee S. Growth patterns and their implications for preterm infants in a culture of rapid modernization. J Child Health Care 2013; 17: 242-52.

[17] Zarrati M, Shidfar F, Moradof M, et al. Relationship between breast feeding and obesity in children with low birth weight. Iran Red Crescent Med J 2013; 15: 676-82.

[18] Yang ZY, Huffman SL. Nutrition in pregnancy and early childhood and associations with obesity in developing countries. Maternal Child Nutri 2013; 9: 105-19.

[19] Camurdan MO, Camurdan AD, Polat S, Beyazova U. Growth patterns of large, small, and appropriate for gestational age infants: impacts of long-term breastfeeding: a retrospective cohort study. J Pediatr Endocr Met 2011; 24: 463-68.

[20] Woo JG, Guerrero ML, Guo F, et al. Human milk adiponectin affects infant weight trajectory during the second year of life. J Pediatr Gastr Nutr 2012; 54: 532-39.

[21] Ong KKL, Ahmed ML, Sherriff A, et al. Cord blood leptin is associated with size at birth and predicts infancy weight gain in humans. J Clin Endocrinol Metab 1999; 84: 1145-48.

[22] Savino F, Liguori SA, Lupica MM. Adipokines in breast milk and preterm infants. Early Hum Dev 2010; 1:77-80.

[23] Walker CD. Maternal touch and feed as critical regulators of behavioral and stress responses in the offspring. Dev Psychobiol 2010; 7: 638-50.

[24] Young L, Morgan J, McCormick FM, McGuire W. Nutrientenriched formula versus standard term formula for preterm infants following hospital discharge. Cochrane Database Syst Rev. 2012; (3): CD 004696.

[25] Boeke CE, Mantzoros CS, Hughes MD, et al. Differential associations of leptin with adiposity across early childhood. Obesity 2013; 21: 1430-37.

[26] Grote V, Schiess SA, Closa-Monasterolo R, et al. The introduction of solid food and growth in the first 2 years of life in formula-fed children: analysis of data from a European cohort study. Am J Clin Nutr 2011; 94: 1785s-93s.

[27] Fanaro $S$. Which is the ideal target for preterm growth? Minerva Pediatr 2010; 62: 77-82.

Received On: 09-July-2014

Accepted On: 07-October-2014

Published On: 23-October-2014

DOI: $10.6000 /$ ijipem/2014/6

() 2014 Litvinchuk et al.; Licensee International Journal of Integrative Pediatrics and Environmental Medicine. This is an open access article licensed under the terms of the Creative Commons Attribution Non-Commercial License (http://creativecommons.org/licenses/by-nc/3.0/) which permits unrestricted, non-commercial use, distribution and reproduction inany medium, provided the work is properly cited. 